\title{
Exposição cianídrica em suínos: Uma abordagem dos parâmetros toxicocinéticos utilizando $o$ tiocianato como biomarcador
}

Helena MANZANO ${ }^{1}$

Altamir Benedito de SOUSA ${ }^{1}$

Silvana Lima GÓRNIAK ${ }^{1}$

\section{Correspondência para:}

Silvana Lima Gómiak

Departamento de Patologia da Faculdade de Medicina Veterinária e Zootecnia da Universidade de São Paulo, Av. Prof. Dr. Orlando Marques de Paiva ${ }^{\circ} 87$ 05508-000 - São Paulo - SP gorniak@usp.br

Recebido para publicação:11/07/2005 Aprovado para publicação: 13/02/2006

1 - Departamento de Patologia da Faculdade de Medicina Veterinária e
Zootecnia da Universidade de São Paulo; São Paulo - SP

\section{Resumo}

O objetivo do presente estudo foi verificar os efeitos sobre a cinética do cianeto, em suínos, em diferentes fases da vida, usando o tiocianato como biomarcador. Vinte e dois suínos, foram divididos em quatro grupos (60 dias da idade, 95 dias da idade, 80 dias do gestação e 21 dias de lactação), e receberam por via oral, a dose única de $3.0 \mathrm{mg} / \mathrm{kg}$ de peso vivo de cianeto do potássio $(\mathrm{KCN})$. As concentrações do tiocianato no sangue foram medidas dentro de $24 \mathrm{~h}$. O tempo máximo (Tmax) e constante de eliminação (Kel) foram mais elevados em porcas lactantes (15 hs e 0.045 , respectivamente); por ouro lado, a maior concentração do tiocianato (Cmax) foi observada nas fêmeas grávidas (161.8). A meia vida de eliminação (t1/2) e o volume da distribuição (Vd) foram mais elevados nas fêmeas adultas (41, 57 e 1.23, respectivamente). Contudo a área sob a curva (AUC) do tiocianato foi mais elevado nos animais novos $(354183,28)$, e o clearance o mais baixo (0.007) nestes animais. Concluindo, os resultados do presente estudo, evidenciam que o metabolismo do cianeto, varia extremamente, considerando o estado fisiológico dos suínos fêmeas, e que são os animais novos, provavelmente, os mais sensíveis aos efeitos tóxicos, da exposição crônica as baixas doses do cianeto.

\section{Introdução}

Humanos e animais consomem, às vezes, substâncias indesejáveis incorporadas às complexas misturas utilizadas como alimento. Em muitos casos, a substância tóxica, aparentemente ausente em um determinado alimento, é formada por ação enzimática sobre um precursor não tóxico, após colheita ou durante a digestão. Os glicosídeos cianogênicos são um bom exemplo destes precursores não tóxicos ${ }^{1}$. A mandioca contém glicosídeos cianogênicos que, em sua hidrólise produzem o ácido cianídrico $(\mathrm{HCN})$, cuja ingestão pode promover intoxicação ${ }^{1}$.

Atualmente, verifica-se um crescente consumo de mandioca nas diferentes espécies animais, particularmente em suínos, onde é consumida na forma in natura, sem ser descascada ${ }^{2}$. No entanto, apesar de apresentar características bastante desejáveis, que preconiza o seu uso na alimentação animal como fornecedor de energia, suplemento protéico e fibras, além de possuir baixo custo para o plantio, bem como resistência a pragas e crescimento em solos pobres e em períodos de seca ${ }^{3,4}$, deve-se considerar que este tubérculo contém glicosídeos cianogênicos, principalmente a linamarina, que pode causar toxicidade, já que a hidrólise deste glicosídeo determina a liberação do cianeto. Neste sentido, vários estudos mostram que as quantidades de cianeto presentes na mandioca podem variar de 10 a $2000 \mathrm{mg} / \mathrm{kg}$, sendo esta grande discrepância encontrada nos níveis desta substância devido à quantidade de glicosídeo cianogênico presente na mandioca cujos níveis, por sua vez, dependerão de alguns fatores, como o tipo de ambiente e as características do solo em que o tubérculo 
foi cultivado, a estação do ano em que foi coletada bem como fatores genéticos relacionados à variedade cultivar ${ }^{5}$.

Os glicosídeos cianogênicos, incluindo a linamarina, são liberados por processos físicos, tais como mastigação, congelação e secagem. Estes processos permitem o contato dos vacúolos, contendo o glicosídeo com uma enzima, encontrada no citosol, denominada b-glicosidase, que proporciona seu desdobramento em açúcar e aglicona, sendo que este e último composto sofrerá ainda ação de mais uma enzima denominada hidroxinitrila-liase que, por sua vez, formará o íon cianeto e um composto aldeído ou cetônico ${ }^{5,6}$. Após a absorção, o cianeto por apresentar alta afinidade pela forma heme férrica da enzima citocromo- oxidase, se liga a esta enzima, inibindo a etapa citocromoa-citocromo-a3, não havendo, assim, a transferência de elétrons na cadeia respiratória, promovendo, portanto, a anóxia histotóxica. Por outro lado, os mamíferos possuem um eficiente mecanismo de detoxificação do cianeto, o qual é realizado por uma enzima denominada acetilsulfotransferase ou rodanase. Esta enzima catalisa a transferência de um átomo de enxofre de um doador, por exemplo, o tiossulfato, para o cianeto, formando principalmente o tiocianato, reação considerada irreversível ${ }^{5}$. O tiocianato, principal metabólito do cianeto, não tem a capacidade de se ligar a citocromo oxidase, sendo eliminado principalmente por meio da urina ${ }^{5,6}$. Portanto, a intoxicação aguda por cianeto ocorrerá somente quando as reações de detoxificação forem excedidas ${ }^{4}$.

Por outro lado, há vários trabalhos na literatura, relatando que a exposição prolongada ao cianeto, em baixas concentrações, pode ocasionar quadros de toxicidade crônica. De fato, em humanos, foi comprovada a associação entre a exposição crônica ao cianeto na etiologia de alguns distúrbios neurológicos, tais como neuropatia atáxica tropical ${ }^{7}$, konzo $^{8}$, neuropatia retrobular da anemia perniciosa ${ }^{9}$ e atrofia óptica de Leber ${ }^{10,11}$. Na criação animal, a ingestão crônica do cianeto tem sido associado a diversas doenças, como a cistite e ataxia enzoótica, uma síndrome caracterizada pela presença de degeneração walleriana da substância branca da medula espinhal e de degeneração de pedúnculos cerebrais e cerebelares ${ }^{12,13,14}$. Uma outra patologia que vem sendo associada à ingestão crônica do cianeto é o bócio. Neste sentido, sabe-se que o principal metabólito do cianeto, o tiocianato, é uma substância bociogênica, já que compete com o iodo na proteína transportadora deste, presente na glândula tireóide, diminuindo a captação de iodo e, conseqüentemente, inibindo a produção dos hormônios tireoideanos ${ }^{15}$. Além disto, tem sido correlacionada a ingestão prolongada de cianeto a lesões renais e hepáticas em coelhos ${ }^{16} \mathrm{e} \operatorname{suínos}^{17} \mathrm{e} \mathrm{a}$ diabetes pancreática, em humanos ${ }^{18}$.

Portanto, como este tipo de intoxicação é de grande interesse, tanto para a criação animal, particularmente suína, bem como em humanos, o conhecimento de sua toxicocinética tem grande relevância, pois é a maneira mais segura e eficaz, com menor relação custo versus benefício, para responder a respeito da margem de segurança de exposição deste agente. Assim foi objetivo deste estudo avaliar a toxicocinética do cianeto, na espécie suína em diferentes fases de vida.

\section{Material e Métodos}

Foram utilizados 22 animais, fêmeas, da espécie suína, provenientes do cruzamento das raças Landrace e Large White, divididos em diferentes grupos por estágios de vida e/ou condição fisiológica, a saber: 6 fêmeas em idade não reprodutiva, com 60 dias de idade, pesando em média $25 \mathrm{~kg} ; 5$ fêmeas, em idade reprodutiva, com 95 dias de idade, pesando ao redor de $45 \mathrm{~kg}$, na fase de diestro; cinco fêmeas prenhes, primíparas, no $80^{\circ}$ dia de gestação, pesando em média $210 \mathrm{~kg}$ e 6 fêmeas lactantes, em diestro, pesando em média $180 \mathrm{~kg}$.

A administração do cianeto de 
potássio $(\mathrm{KCN})$ foi feita através da via oral, juntamente com a ração, pela manhã (8:00h), após jejum prévio de 12 horas, na dose única de $3,0 \mathrm{mg} / \mathrm{kg}$ de peso vivo. A coleta de sangue foi feita por punção da veia carótida externa, em seringas descartáveis não heparinizadas, em intervalos de tempo, dentro de um período de 24 horas. Após este procedimento, este material biológico foi centrifugado por $15 \mathrm{~min}$ a $3000 \mathrm{rpm}$ e o soro acondicionado individualmente em microtubos plásticos (EppendorfÒ), devidamente identificados e estocados em congelador a $-20^{\circ} \mathrm{C}$ até o processamento. A determinação do tiocianato seguiu a metodologia proposta por Pettigrew e Feell ${ }^{20}$, modificada em nosso laboratório.

Os dados são apresentados na forma de média, seguida por seu respectivo erro padrão. Foi empregada a análise de variância ANOVA, proposta por Snedcor ${ }^{21}$, seguida do teste de Dunnett ${ }^{22}$ para a detecção das diferenças significantes entre os grupos. A probabilidade de $\mathrm{p}<0,05$ foi considerada capaz de revelar diferenças significantes entre os grupos. Em relação aos dados toxicocicénticos, o pico de concentração máxima (Cmax) do tiocianato nos meios biológicos estudados e o tempo para atingir este pico (Tmax), foram obtidos diretamente dos dados experimentais sem interpolação dos mesmos. A área sobre a curva (ASC) foi calculada pelo método dos trapezóides lineares. Todos os outros parâmetros toxicocinéticos foram calculados como descrito por Shargel e Yu ${ }^{23}$.

\section{Resultados}

Nenhum dos animais, pertencentes aos diferentes grupos, apresentaram qualquer sinal de intoxicação cianídrica aguda.

A figura 1 ilustra os resultados obtidos nas dosagens dos níveis séricos de tiocianato de suínos fêmeas, com diferentes estágios de vida, imediatamente antes $(\mathrm{t}=0)$, da administração de $3,0 \mathrm{mg} / \mathrm{kg}$ de $\mathrm{KCN}$, por via oral, junto com ração comercial e após este procedimento, em horários distintos.Assim, pode-se verificar que houve aumento significante $(\mathrm{p}<0,05)$ nas concentrações séricas de tiocianato a partir dos 30, 60, 180 e 360 min., após a administração do cianeto, naquelas fêmeas pré-puberes, adultas, gestantes e lactantes, respectivamente. Em todos estes animais, o aumento significante de tiocianato persistiu até a $24^{a}$ hora (ou $21^{a}$ hora naquelas fêmeas gestantes), após a administração do $\mathrm{KCN}$. $\mathrm{Na}$ tabela 1 são apresentados os parâmetros toxicocinéticos dos grupos estudados.

\section{Discussão}

Devido a razões econômicas, a mandioca vem sendo cada vez mais usada como substituinte do milho na alimentação animal, particularmente na de suínos ${ }^{24}$. Como conseqüência, pode-se supor que estes animais podem estar expostos a baixos, mas constantes níveis de cianeto, por períodos prolongados. A dose de cianeto escolhida para ser administrada no presente estudo $(3,0 \mathrm{mg} / \mathrm{kg})$ está relacionada aos níveis de cianeto, os quais os animais são passíveis de serem naturalmente expostos, quando consumindo alimentos com glicosídeos cianogênicos, como, por exemplo, a mandioca ${ }^{24}$.

A via oral é a principal via de exposição a agentes tóxicos, tanto para humanos como para animais ${ }^{25}$; além disto, sabe-se que a propagação de xenobióticos se faz, preferencialmente, por esta via, por meio de ingestão de água e/ou do alimento contaminado ${ }^{26}$. Ainda, deve ser considerado que a administração por via oral vem sendo amplamente utilizada em toxicologia, devido à conveniência, baixo custo e mínimo estresse para o animal ${ }^{26}$. Portanto, na presente pesquisa, optou-se por esta via de administração.

Os dados aqui obtidos revelaram que nenhum dos animais, pertencentes aos diferentes grupos experimentais, mostraram sinais de intoxicação aguda pelo cianeto e que variações no comportamento da curva versus tempo, em relação aos níveis séricos de tiocianato, foram significantes para todos os grupos, quando se comparou com 
aqueles dados obtidos, destes mesmos animais, antes da administração do KCN.

A cinética de uma substância química no organismo pode ser bastante complexa, já que vários processos, como a absorção, a distribuição e a eliminação, atuam alterando as concentrações nos tecidos e líquidos biológicos. Por outro lado, estes processos variam devido a diversos fatores como espécie animal, idade e sexo, entre outros ${ }^{27}$. De fato, um estudo realizado neste laboratório, no qual se comparou osparâmetros cinéticos do tiocianato, entre trêsespécies animais: caprina, suína e ratos, foi possível verificar claramente que o metabolismo do cianeto está diretamente relacionado à espécie animal ${ }^{28}$.

Além disto, um outro aspecto que deve ser considerado é a variação na cinética de absorção, biotransformação e eliminação de uma determinada substância em fêmeas; neste sentido, é amplamente conhecido que os dados referentes ao metabolismo de um determinado xenobiótico são díspares quando se compara a exposição ao mesmo em fêmeas, em diferentes estados fisiológicos, tais como antes da puberdade, na idade adulta, na gestação e na lactação ${ }^{29}$. Assim, foi objetivo deste estudo verificar se, em relação à cinética do cianeto, os parâmetros toxicocinéticos também a presentar-se-iamdiscrepantes, considerando-se fêmeas, da espécie suína, em diferentes fases fisiológicas.

De fato, a comparação entre os dados dos níveis séricos de tiocianato permitiu verificar que, apesar do comportamento da curva relativa à concentração de tiocianato ao longo do tempo ter sido semelhante entre os distintos grupos, o período de tempo no qual se iniciou o aumento significante dos níveis séricos deste metabólito foi diferente para cada grupo, sendo que os dados mais discrepantes referem-se àqueles obtidos das fêmeas pré-púberes, cujo aumento significante já foi observado $30 \mathrm{~min}$. após a exposição ao $\mathrm{KCN}$ e aquele das porcas lactantes, que apresentaram esta elevação dos níveis de tiocianato somente na $6^{a}$ hora após a ingestão do cianeto. Duas hipóteses poderiam ser levantadas para explicar esta grande diferença encontrada, a primeira estaria relacionada ao $\mathrm{pH}$ e $\mathrm{pKa}$; neste sentido, sabe-se que um conhecido fator que altera a absorção de uma determinada substância, quando administrada por via oral, é a relação do pKa desta substância e o pH do ambiente gastrintestinal. Como o cianeto é um ácido fraco, com pKa de 9,22 $2^{30}$, poderia-se supor que haveria diferenças no $\mathrm{pH}$ do estomago destes animais, conforme o estado fisiológico. Portanto, seguindo-se este raciocínio, seria possível inferir que o compartimento gástrico de fêmeas prépuberes teriam o $\mathrm{pH}$ mais acídico que aquelas pertencentes aos outros grupos, o que permitiria gerar maior quantidade de moléculas não-ionizadas, acarretando em maior eficácia na absorção do cianeto, e o contrário ocorreria com aquelas fêmeas lactantes.

A outra hipótese que poderia explicar esta discrepância e, talvez, a mais provável, está relacionada à velocidade de formação do tiocianato. Assim, estima-se que $80 \%$ do cianeto absorvido é transformado em tiocianato, pela enzima rodanase ${ }^{31}$ (ou acetilsulfotransferase), sendo, então, este produto praticamente todo eliminado por via renal ${ }^{31}$. Portanto, é possível supor que estas diferentes condições fisiológicas possam determinar variações nos níveis da enzima rodanase, o que acarretaria em maior ou menor eficiência na formação do tiocianato.

A análise dos dados relativos aos níveis séricos de tiocianato mostrou ainda, quando da comparação entre os valores obtidos dos diferentes grupos de fêmeas, que os animais gestantes tiveram, de maneira geral, níveis de tiocianato superiores quando comparados com aquelas fêmeas não prenhes e lactantes. Uma possível explicação para este fato poderia estar correlacionada à transformação do cianeto em tiocianato pela placenta, já que se sabe que este órgão possui quantidades elevadas da enzima rodanase ${ }^{32}$. Desta maneira, a placenta seria um local adicional 
para a formação do metabólito do cianeto. Corrobora com esta suposição os dados relativos ao $\mathrm{C}_{\max }$, parâmetro que indica $\mathrm{o}$ pico da concentração sérica de qualquer substância, neste caso do tiocianato; portanto, quanto maior o valor $\mathrm{da} \mathrm{C}_{\max }$, maior será a taxa de transformação do cianeto em tiocianato, cujos valores mais altos foram encontrados justamente naquelas fêmeas gestantes. Um outro dado obtido da cinética, que reforça esta suposição, refere-se ao $T_{\max }$, ou seja, o parâmetro que indica o tempo no qual ocorre o pico de concentração sérica, e que, neste estudo, foram as fêmeas prenhes aquelas que apresentaram este tempo mais precocemente (6 hs) em relação aos outros grupos.

Por outro lado, aceitando-se tal conjetura, seria esperado que o aumento significante nos níveis de tiocianato nas marrãs gestantes fosse também o mais precoce que de outras fêmeas nos diferentes estados fisiológicos e não o de fêmeas prépuberes, como já discutido. Contudo, devese considerar que há redistribuição do tiocianato nos órgãos ou líquidos corporais destas fêmeas, bem como a passagem do cianeto para o feto, pois, substâncias aniônicas, tais como ácidos fracos, podem se acumular no embrião, possivelmente devido ao gradiente de $\mathrm{pH}$ entre o plasma materno, que é mais ácido em relação ao $\mathrm{pH}$ do plasma fetal, tornando este composto na forma ionizável no embrião. Portanto, supõe-se que embora havendo um aumento na capacidade de biotransformação do cianeto por fêmeas gestantes, num primeiro momento pode ter havido um "aprisionamento" do cianeto no feto, o que levaria ao retardado este processo.

Ainda, avaliando-se a redistribuição do tiocianato nos diferentes compartimentos e líquidos corporais, deve-se considerar em especial a passagem deste metabólito para o leite. Assim, estudos recentes mostram que na glândula mamária existe uma proteína denominada de $\mathrm{Na}^{+} \mathrm{I}^{-}$symporter (NIS), que desempenha a mesma função apresentada na tireóide, ou seja, o transporte de iodeto para o leite ${ }^{34}$, já que esta substância seria uma fonte supridora de iodeto para o neonato. Portanto, é possível supor que, analogamente ao que ocorre na tireóide, o tiocianato poderia estar competindo com o iodeto, sendo, então excretado em grande quantidade para o leite. Concorda com esta suposição aqueles estudos realizados neste laboratório, os quais mostraram que ratas lactantes intoxicadas com KCN apresentam níveis elevados de tiocianato no leite ${ }^{35}$. De fato, embora tal dosagem não tenha sido realizada neste estudo, no leite das porcas, foi possível observar que estas fêmeas foram aquelas que apresentaram maior valor de constante de eliminação (kel), menor área sobre a curva (ASC), bem como meia-vida de eliminação (T1/2b).

Considerando-se o volume de distribuição $(\mathrm{Vd})$, verificou-se que fêmeas com 95 dias de idade foram aquelas que apresentaram os maiores valores. Como este parâmetro expressa a extensão da distribuição da substância fora do plasma e dentro de outros tecidos, pode-se inferir que o tiocianato apresentou maior penetração nos tecidos destas fêmeas. Uma vez que animais nesta faixa de idade se encontram na fase de terminação, supõe-se que estas marrãs tenham maior proporção nos níveis de gordura corporal que o de outros animais em outros estados fisiológicos. Portanto, pode-se especular que esteja havendo a redistribuição do tiocianato para o tecido adiposo; no entanto, futuros estudos relativos à avaliação do rendimento de carcaça, deverão ser conduzidos para melhor alicerçar esta teoria.

A análise dos demais parâmetros toxicocinéticos mostrou que os maiores valores da ASC obtidos naquelas fêmeas prépúberes. Cabe ressaltar aqui que estas fêmeas encontravam-se ainda na creche, sendo, portanto, consideradas muito jovens. Uma vez que a ASC é inversamente proporcional ao clearance $^{36}$, e sendo este último parâmetro, por sua vez, atrelado à eliminação do tiocianato, pode-se concluir que, de fato, a excreção deste metabólito em animais nesta 

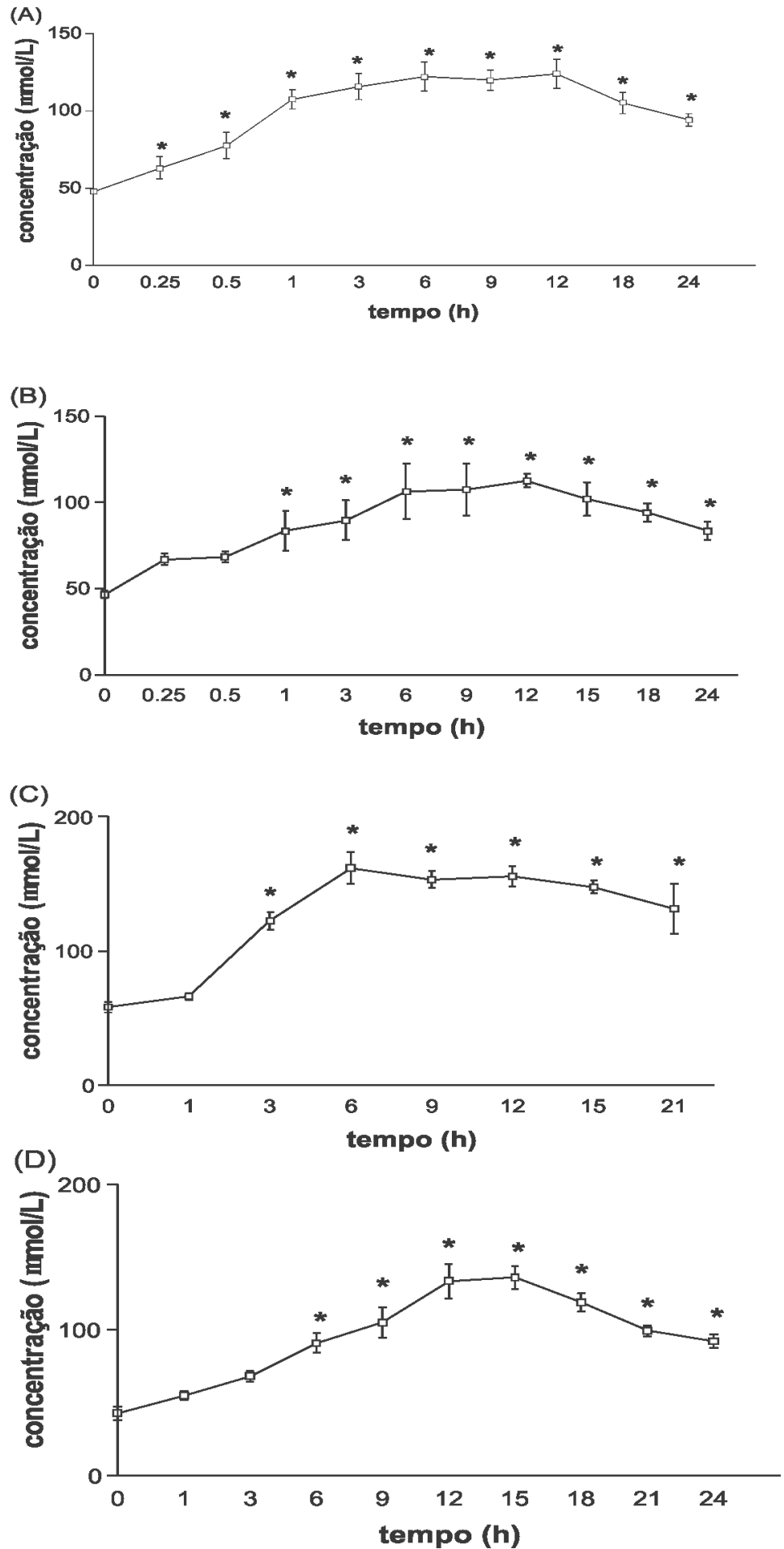

Figura 1 - Níveis séricos de tiocianato, em umol/L, em suínos fêmeas com; 60dias (A) e 95dias (B) de idade, 80dias de gestação (C), e 21 dias de lactação $\quad$ (D), antes e após a administração de $3,0 \mathrm{mg} / \mathrm{Kg}$ de cianeto de potássio (KCN), por via oral, junto com ração comercial 
Tabela 1 - Dados dos estudos toxicocinéticos do cianeto de potássio $(\mathrm{KCN})$ em fêmeas da espécie suína, nas diferentes fases de vida: jovens com 60 dias de idade, adultas com 95 dias de idade, e em diestro, adultas com 80dias de gestação, adultas com 21dias de lactação., que receberam, por via oral, a dose de 3,0mg/Kg de KCN, juntamente com ração comercial. (Cmax: concentração máxima; Tmax: tempo para atingir a Cmax; ASC: área sob a curva; Kel; constante de eliminação; Cl: clearance, t1/2 $\beta$ :meia vida de eliminação; Vd: volume aparente de distribuição. Pirassununga 2005

\begin{tabular}{|c|c|c|c|c|}
\hline \multicolumn{5}{|c|}{ Grupos } \\
\hline $\begin{array}{l}\text { Parâmetros } \\
\text { toxicocinéticos }\end{array}$ & $\begin{array}{l}\text { 60dias de } \\
\text { idade }(n=6)\end{array}$ & $\begin{array}{c}\text { 95dias de } \\
\text { idade }(n=5)\end{array}$ & $\begin{array}{c}\text { 80dias de } \\
\text { gestação } \\
(n=5)\end{array}$ & $\begin{array}{l}21 \text { dias de } \\
\text { Lactação } \\
(n=6)\end{array}$ \\
\hline Cmax (mcmol/L) & 124,3 & 112,7 & 161,8 & 136,0 \\
\hline $\operatorname{Tmax}(\mathrm{h})$ & 12 & 12 & 6 & 15 \\
\hline $\operatorname{Kel}\left(h^{-1}\right)$ & 0,019 & 0,017 & 0,019 & 0,045 \\
\hline $\mathrm{Cl}(\mathrm{L} . \mathrm{Kg} / \mathrm{h})$ & 0,007 & 0,020 & 0,016 & 0,019 \\
\hline$T I / 2 \beta$ & 37,26 & 41,57 & 36,21 & 15,47 \\
\hline$V d(L / k g)$ & 0,38 & 1,23 & 0,82 & 0,42 \\
\hline 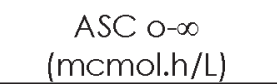 & $354.183,28$ & $140.513,12$ & $158.834,34$ & $57.080,61$ \\
\hline
\end{tabular}

faixa etária é menos eficiente que daquelas fêmeas em outras condições fisiológicas, avaliadas neste estudo, já que a maturação do tecido renal é fundamental para que ocorra a eficiente eliminação do metabólito do cianeto, já que esta se faz quase que exclusivamente através da urina ${ }^{31}$. Este dado concorda com um dos importantes conceitos em toxicologia, o qual preconiza que, de maneira geral, animais jovens são mais susceptíveis aos efeitos tóxicos de uma substância ${ }^{27}$.

Concluindo, a presente pesquisa evidencia claramente que há grandes diferenças no perfil toxicocinético do cianeto, quando se considera as distintas fases fisiológicas da fêmea. Além disto, os dados aqui obtidos permitem sugerir serem as fêmeas pré-púberes aquelas mais sensíveis aos efeitos tóxicos produzidos pela exposição crônica ao cianeto.

\section{Cianidric exposition in swines: A bording of the toxicocinetics parameters using the tiocianato as biomarker}

\section{Abstract}

The aim of the present study was to determine the effects of the swine, in different periods of life on the toxicokinetics of cyanide using thiocyante as biomaker. Twenty and two swines, was divided into four groups ( 60 days of age, 95 days of age, sows with 80 days of gestation and lactating swine), were dosed orally with $3,0 \mathrm{mg} / \mathrm{kg} /$ body weigth of potassium cyanide $(\mathrm{KCN})$. Thiocyanate concentrations in blood were measured within $24 \mathrm{~h}$. The time of peak concentration (Tmax) and constant of elimination (Kel) were higher in lactating sows ( $15 \mathrm{hs}$ and 0,045 , respectively); on the other hand, the maximum plasma concentration (Cmax) of thiocyanate was observed in pregnant females $(161,8)$. The elimination half life $(\mathrm{t} 1 / 2)$ and volume of distribution $(\mathrm{Vd})$ were higher in adult sows $(41,57$ and 1,23, respectively). Whereas the clearance and the area under the curve (AUC) of thiocyanate was higher in young animals $(354183,28)$ the clearance was lower $(0,007)$ in these animals. In coclusion, the results of the present study evidence that the metabolism of cyanide varies greatly
Key-words

Cyanide.

Thiocyante.

Toxicokinetics.

Swine. 
considering the physiologic state of female swine being the young animals probably more sensitive to the toxic effects of chronic exposure to low doses of cyanide.

\section{Referências}

1 TELES, F. F. Técnicas de liberação do HCN e toxidez cianogênica das mandiocas. Informe Agropecuário, v. 13, n. 145, p. 18-19, 1987.

2 OKE, O. L. The use of cassava as pig feed. Nutrition Abstract and Reviews- series B. Commonweath bureau of nutrition, v. 54, n. 7, p. 301-314, 1984

3 HENRY,G.; CORREA, C. Economics of cassava product use in animal feeding. Food and Agriculture Organization of United Nations, Rome-FAO, v. 95, p. 183-196, 1992.

4 OKE, O. L. Toxicity of cyanogenic glicosides. Food Chemistry, v. 8, p. 97-109, 1980.

5 ONG, H. K. Cianogenic and goitrogenic factors in pig nutrition. Pig News an information, v. 10, n. 1, p. 19-23, 1989.

6 POULTON, J. E. Cyanogenic compounds in plants and their toxics effects. In: KEELER, R. F.; TU, A. T. (Ed.). Handbook of natural toxins: plant and fungal toxins. New York: Marcel Dekker, 1983. v. 1, p. 117 157.

7 PANIGRAHI, S.; RICHARD, J.; O'BRIEN, G. M.; GAY, C. Effects of different rates of drying cassava root on its toxicity to broiler chicks. British Poultry Science, v. 33, n. 5, p. 1025-1042, 1992.

8 OSUNTOKUN, B. O Ataxic neuropathy associed with hith cassva diets in West África. In: NESTEL, B.; MACINTYRE, R. (Ed.), Chronic toxicity. Otawa: International Devolupment Research Center, 1973. p. 127-136.

9 TYLLESKÄR, T.; BANEA, M.; BIKANGI, N.; NAHIMANA, G.; PERSSON, L. A.; ROSLING, $\mathrm{H}$. Dietary determinants of a non-progressive spastic paraparesis (KONZO): a case-referent study in a high incidence area of Zaire. International Journal of Epidemioly, v. 24, n. 5, p. 949-956, 1995.

10 FREEMAN, A. G. Opitic neuropathy and chronic cyanide intoxication: a review. Journal of the Royal Society of Medicine, v. 81, n. 2, p. 103-106, 1988.

11 SYME, L. G.; BRONTE-STEART, J.; FOULDS, W. S.; MACCLURE, E.; LOGAN, R.; STANSFIELD, M. Clinical and Biochimistry findings in Leber's hereditary atrophy. Transactions of the Ophthalmological Society of United Kingdom, v. 103, n. 5, p. 556-559, 1983.

12 TSAO, K.; AIKEN, P. A.; JOHNS, D. R. Smoking as an etiological factor in a pedigree with Leber's hereditary. British Journal of Ophtalmology, v. 83, n. 5, p. 577-581, 1999.
13 ADAMS, L. G.; DOLLAHITE, J. W.; ROMANE, W. M.; BULLARD, T. L.; BRIDGES, C. H. Cystitis and ataxia associated with sorghum ingestion by horses. Journal of American Veterinarian Medicine Association, v. 155, p. $518-524,1969$.

14 MCKENZIE, R. A.; MCMICKING, L. I. Ataxia and urinary incontinence in cattle grazing sorghum. Australian Veterinary Journal, v. 53, p. 496-497, 1977.

15 BRADLEY, G. A.; METCALF, H. C.; REGGIARDO, C.; NOON, T. H.; BICKNELL, E. J.; LOZANOALARCON, F.; FREED, R. E.; RIGGS, M. W. Neuroaxonal degeneration in sheep grazing sorghum pasture. Journal of Veterinary Diagnostic Investigation, v. 7, p. 229-236, 1995

16 KANEKO, J. J. Thyroid Function. In: KANEKO, J. J. Clinical biochesmitry of domestic animals, $4^{\mathrm{a}}$. ed. San Diego: Academic Press, 1989. p. 630-649.

17 OKOLIE, N. P.; OSAGIE, A. U. Liver and Kidney Lesions and Associated Enzyme changes induced in rabbits by chronic cyanide exposure; Food and Chemical Toxicology, v. 37, n. 7, p.745-750, 1999.

18 MANZANO, H. Avaliação da toxicidade do cianeto em suínos durante a fase de crescimentoà terminação: aspectos bioquímicos e anatomopatológicos. 2001. 1224 f. Dissertação (Mestrado) - Faculdade de Medicina Veterinária e Zootecnia, Universidade de São Paulo, São Paulo, 2001.

19 KAMALU, B. P. The adverse effects of long term cassava (Manihot sculenta Grantz) consumption. International Journal of Food Science Nutrition, v. 46, n. 3, p. 65-93, 1995.

20 PetTigReW, A. R.; FELL, G. S. Simplified colorimetric determination of thiocyanate in biological fluids and its applications to investigation on the toxic amblyopias. Clinical Chemistry, v. 18, p. 996-1000, 1972.

21 SNEDCOR, G. W. Statistical methods. $4^{\text {th }}$ ed. Ames: College Press, 1946. 324 p.

22 SPIEGEL, M. R. Estatística. 2 ed. São Paulo: McGrawHill, 1985. p. 233-248.

23 SHARGEL, L.; YU, A. B. C. Applied biopharmaceutics in teratogenics. Florida: CRG Press, 1987. p. $72-76$.

24 MORENO, E. H. Mandioca, potencial energético na alimentação do suíno. Londrina-PR: Fundação Instituto Agronômico do Paraná, 1982. p. 53. (Circular, n. 27)

25 WAY, J. L. Cyanide intoxication and its mechanism of antagonism. Annual Review Pharmacology and Toxicology, v. 24, n. 2, p. 451-481, 1984. 
26 YUAN, J. H. Modeling blood/plasma concentration in dosed feed and dosed drinking water toxicology studies. Toxicology and Applied Pharmacology, v. 119, n. 2, p. 131-141, 1992.

27 MEDINSNKY, A. M.; VALENTINE, L. J. Toxicokinets. In: KLAASEN, C. D. Casarret e Doull's toxicology: the basic neg, science of paisons. $6^{\text {th }}$ ed., Cidade: Editora, 2001. p. 225-238.

28 SOUSA, A. B.;MANZANO, H.; SOTO-BLANCO, B.; GORNIAK, S. L. Toxicokinetics of cyanide in rats, pigs and goats after oral dosing with potassium cyanide. Archives of toxicology, v. 77, n. 6, p. 330-334, 2003.

29 JOCHMANN, N.; STANGLE, K.; GARBE, E.; BAUMMANN, G.; STANGL, V. Female-specific aspects in the pharmacotherapy of chronic cardiovascular diseases. European Heart Journal, 2005, no prelo.

30 CALIAMANIS , A.; MCCORMIK M.J.; CARPENTER, P.D. Enhanced conductometric detector of cyanide in suppressed ion chromatography. Journal Chromatography A, 884, p. 75-80, 2000.

31 ANSELL, M.; LEWIS, F. A. A review of cyanide concentrations found in human organs. A survey of literature concerning cyanide metabolism, 'normal', non-fatal and fatal body cyanide levels. Journal of Forensic Medicine, v. 17, n. 4,p. 148-155, 1970.

32 TEWE, O. O.; MANER, J. H.; GOMEZ, G. Influence of cassava diets on placental thiocyanate transfer, tissue rhodanase activity and performance of rats during gestation. Journal of Science of Food and Agriculture, v. 28, n. 8, p. $750-756,1977$.

33 DENCKER, L.; DANIELSON, B. R. G. Transfer of drugs to the embryo and fetus afther placentation. In NAU, H.; SCOTT, W.J.(Ed). Pharmacokinetics in teratogenics. Florida: CRC Press, 1987, p. 56-67.

34 SPITZWEG, C.; JOBA, W.; SCHRIEVER, K. ; GOELLNER, J. R.; MORRIS, J. C.; HEUFELDER, A. E. Analysis of Human Sodium lodide Symporter Immunoreactivity in Human Exocrine Glands. The Journal of Clinical Endocrinology \& Metabolism, v. 84, n. 11, p. 4178-4184, 1998.

35 SOUSA, A. B. Avaliação dos efeitos tóxicos do cianeto e do tiocianato no período perinatal. Estudo em ratos. 2004, $224 \mathrm{f}$. Tese (Doutorado) - Faculdade de Medicina Veterinária e Zootecnia, Universidade de São Paulo, São Paulo.

36 VRIES, J. Toxicokinets- quantitative aspects.In: NIESSINK, R. J. M.; VRIES, J. HOLLINGER, M. A. (Ed.) Toxicology principles and applications. New Yok: CRC Press, 1996. p. 137-183. 